\title{
Analysis of Mobile Terminal Monitoring System in Greenhouse Based on Internet of Things Technology
}

\author{
Lingling Zheng ${ }^{1, a}$, Wen $\mathrm{Li}^{2, \mathrm{~b}}$ \\ ${ }^{1}$ Jiangsu University ,No.301 Xuefu Road,Jingkou District,Zhenjiang City,Jiangsu Province \\ ${ }^{2}$ Jiangsu University ,No.301 Xuefu Road,Jingkou District,Zhenjiang City,Jiangsu Province \\ A15751003489@163.com, bw@mail.ujs.edu.cn
}

\section{Keywords: greenhouse monitoring system IOT Android}

Abstract: In view of the development of greenhouse and greenhouse monitoring system at home and abroad, the traditional manual control mode has been unable to meet the production demand. Now the Internet of Things technology and greenhouse system combined, proposed based on the Android operating system, intelligent monitoring. The environmental parameters of the crop growth process are recorded and analyzed by the Internet of Things sensing technology. According to the best suitable range of each factor, the automatic control of the mobile terminal interface is carried out to ensure the product quality and product quantity of the crop. Combined with the characteristics of quality sensing frame data collection characteristics and crop growth environment impact factor characteristics, put forward the monitoring system requirements, and conduct system requirements design.

\section{Introduction}

In order to deal with the increasingly harsh environment, and at the same time in order to solve the problem of uneven population and quantity of grain supply and demand, the national level of greenhouse technology proposed intelligent, automated development needs, domestic and foreign research scholars have combined with emerging Internet technology, Greenhouse monitoring system. Through the site to collect the environmental parameters, combined with crop growth status to determine the appropriate environmental status, the light value, moisture content control. At present, most of the domestic greenhouse monitoring system to achieve the regional environmental regulation, can not guarantee that each crop to get the most suitable environment, so how accurate to each crop fine tuning is a key issue to be solved.

In 2007, the intelligent development of the terminal equipment driven by mobile, mobile intelligent terminal instantly replace the traditional computer terminals to become the Internet industry-related industries and platforms. Based on mobile intelligent terminal monitoring system has a strong flexibility and immediacy, can achieve 24-hour remote control. This paper presents the "Internet of Things + Greenhouse + Android" three-dimensional intelligent monitoring system for the realization of crop growth process precision, automation, implementation of management and control.

\section{Greenhouse monitoring system overall framework}

"Internet of Things + greenhouses + Android" three-dimensional intelligent monitoring system to the Internet of things as the front, through the GPRS and other sensors to collect greenhouse greenhouse on-site crop environmental data and growth data; in the server-side data integration and analysis, the Data transmission to the terminal control interface; terminal interface can query each plant data information, by clicking the device button to achieve remote control of the scene environment.

The field control center is equipped with control equipment, alarm equipment and video surveillance. Field control center mainly to complete data management, intelligent decision-making, historical data analysis, and data display, editing, storage function. Can be monitored according to the monitoring 
system software interface to carry out real-time intelligent control or notify the scene of the staff to carry out manual control to ensure that crops have a good environment for growth

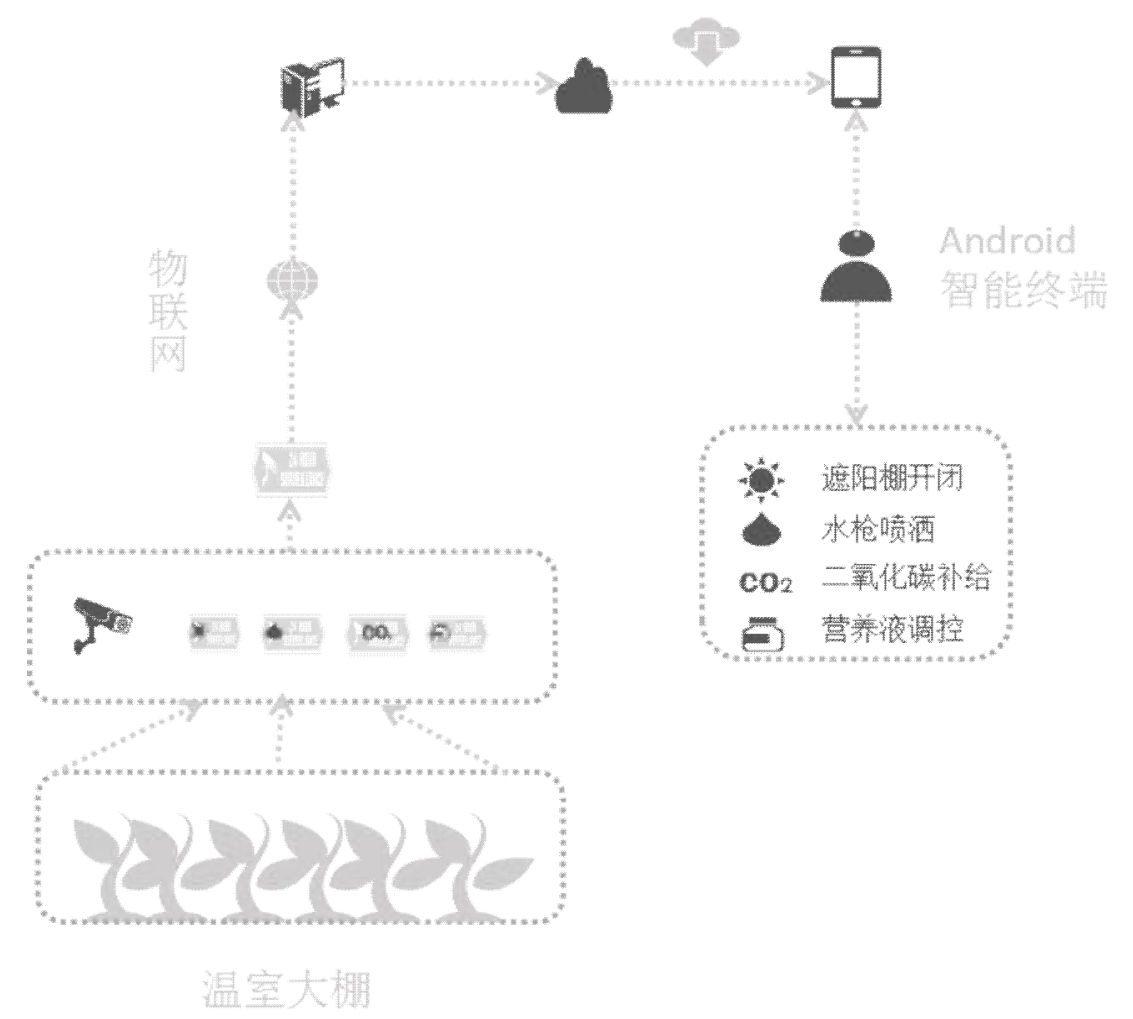

Fig. 1 greenhouse greenhouse monitoring system overall structure

\section{Internet of Things technology}

Through the Internet of things technology, you can build greenhouse data collection, real-time transmission, intelligent processing and monitoring system. The use of sensor technology, the target crop identification, to ensure high speed, high efficiency, high storage acquisition. The sensor is used to detect the light, moisture, $\mathrm{CO} 2$ and nutrient content of the environment, and the original data are screened and transmitted to the terminal by self-organizing wireless communication network.

Aiming at the large number of greenhouse data and the characteristics of a large number of greenhouse, we propose to deal with massive heterogeneous data using the mass sensing network. Using the blend of perception and service modules will be different sources of data integration, standardization. Increase the semantic annotation layer and data storage layer, and realize the comprehensive and efficient collection of greenhouse data. Most of the domestic greenhouses with small scattered non-characteristics, the framework not only to ensure that the same greenhouse data within the different types of unified integration, but also to ensure the effective integration of different data within the greenhouse. Privacy protection middleware to ensure that data transmission and use of the process, special information can only be provided to specific user inquiries for the follow-up retrograde greenhouse system to provide possible.

Internet of things will be processed after the initial transfer to the cloud platform, the cloud is the monitoring system of the server. Cloud as the control center of the system, including the coordination of data, transmission of data, pooling data and other functions, through the understanding of wireless networks and mobile intelligent terminal pairing. Pairing authentication, the cloud will be the user needs to query the information and issued control instructions were downloaded and uploaded. 
Android is based on Linux, local open source features, applied to the mobile terminal device operating system. With a high degree of openness, reusability, comfortable from the operators, and Google applications seamless combination of the five major advantages; at the same time because of its easy operation, the development of simple and rapid occupation of smart phones $75 \%$ market. In the mobile traffic and wireless network development, the establishment of mobile intelligent terminal monitoring system, from geographical and time control, to meet a wide range of user needs. At the same time, the monitoring system is different from the traditional management system, not only to achieve the query and monitoring functions, but also through the interface to achieve remote control equipment, to achieve timely timeliness.

\section{monitoring system components}

\section{Business process analysis}

Based on the actual situation of domestic crop cultivation and the field investigation, the process of crop cultivation was analyzed in detail. The greenhouse environment monitoring process, including the growth of crops in the state of the record, that is, the growth cycle, the growth of normal or not; the real-time environment to measure the crop environmental parameters and their growth needs match; the environment regulation, when the environment does not match, Control the device switch to control.

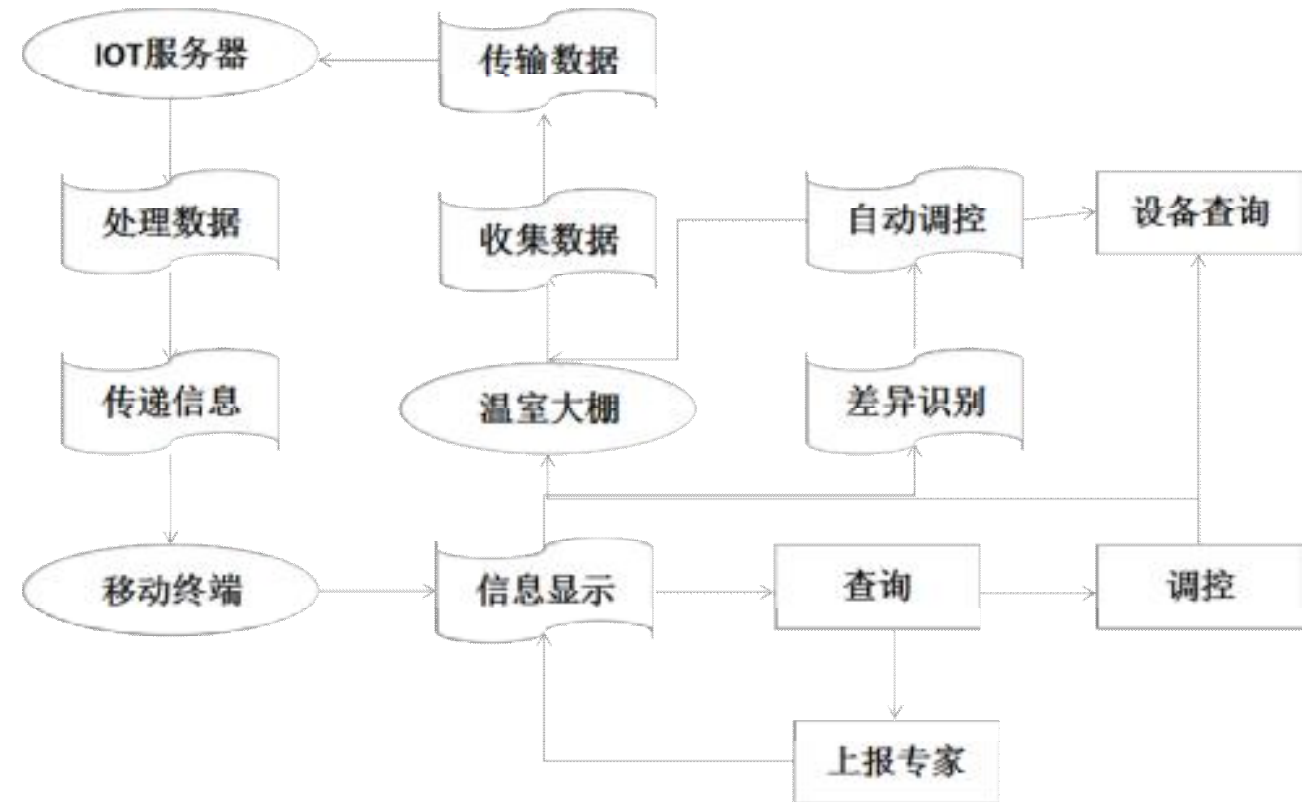

Fig.2 monitoring system business flow chart

Monitoring system business is based on the realization of the Internet of Things technology from the greenhouse collection of data collected, the accuracy of the data determinism control the correctness. The regulation of the system includes both artificial and automatic methods. Artificial research is the focus of the study. The difference recognition process is based on the fuzzy algorithm and the neural network to summarize the crop growth process. Store the system, the system will be based on crop species, the growth phase to read the corresponding value, which is collected with the data automatically compared, and the comparison of the numerical differences will be fed back to the control of each device amplitude.

\section{Data flow analysis}

The monitoring system mainly involves data including field environment data, growth status data, and standard environment values. The three data from the sensor and artificial channels were transferred 
to the monitoring system database. The user can query and call the above data through the mobile terminal control interface.

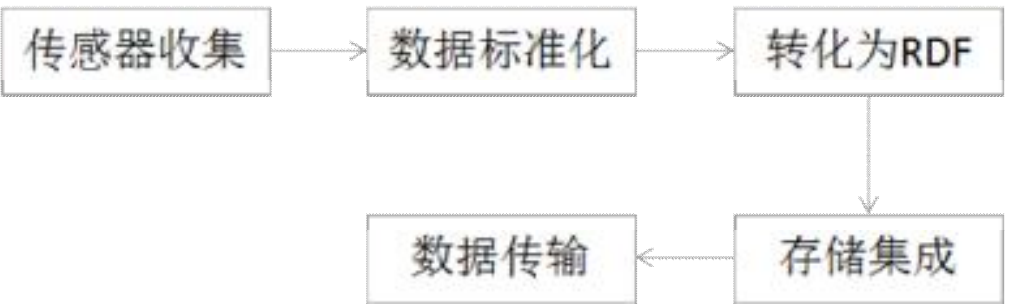

Fig.3 Quality-aware frame data processing

As a result of the integration of the Internet of Things quality perception framework, the monitoring system's data collection consists of roughly five processes. In the semantic annotation layer, the same data model is used to standardize the different types of data captured by the same data model. After the storage layer is integrated, the data is collected by the network layer. Data transmission.

In order to further improve the accuracy of regulation, the type of data collection includes not only physical quantity, light intensity (lumen $/(\mathrm{lm})$ ), moisture percentage, ph value size, etc., also includes the monitor video and audio. Users can observe the video by observing the video, and also understand the quality of the crop. Currently, on the Android platform, you can use MediaPlayer and VideVoview to decode the video to get calls. The video data complements the physical integrity of the fabric, allowing for a more vivid crop virtual model in the control interface.

video hardware decoding. In the Android platform, the default decoding of the video format there are two, namely $\mathrm{mP} 4$ format and $3 \mathrm{gp}$ format. It can be through MediaPlayer and VideoView two ways to video decoder to a certain degree of call.At present, the more popular way is through the transplant FFmPeg open source library to achieve H.264 format video decoding.In the way of receiving the picture, the video decoding function is mainly implemented by the server side, so the Android client only needs to receive the decoded image data effectively.

\section{前台功能图}

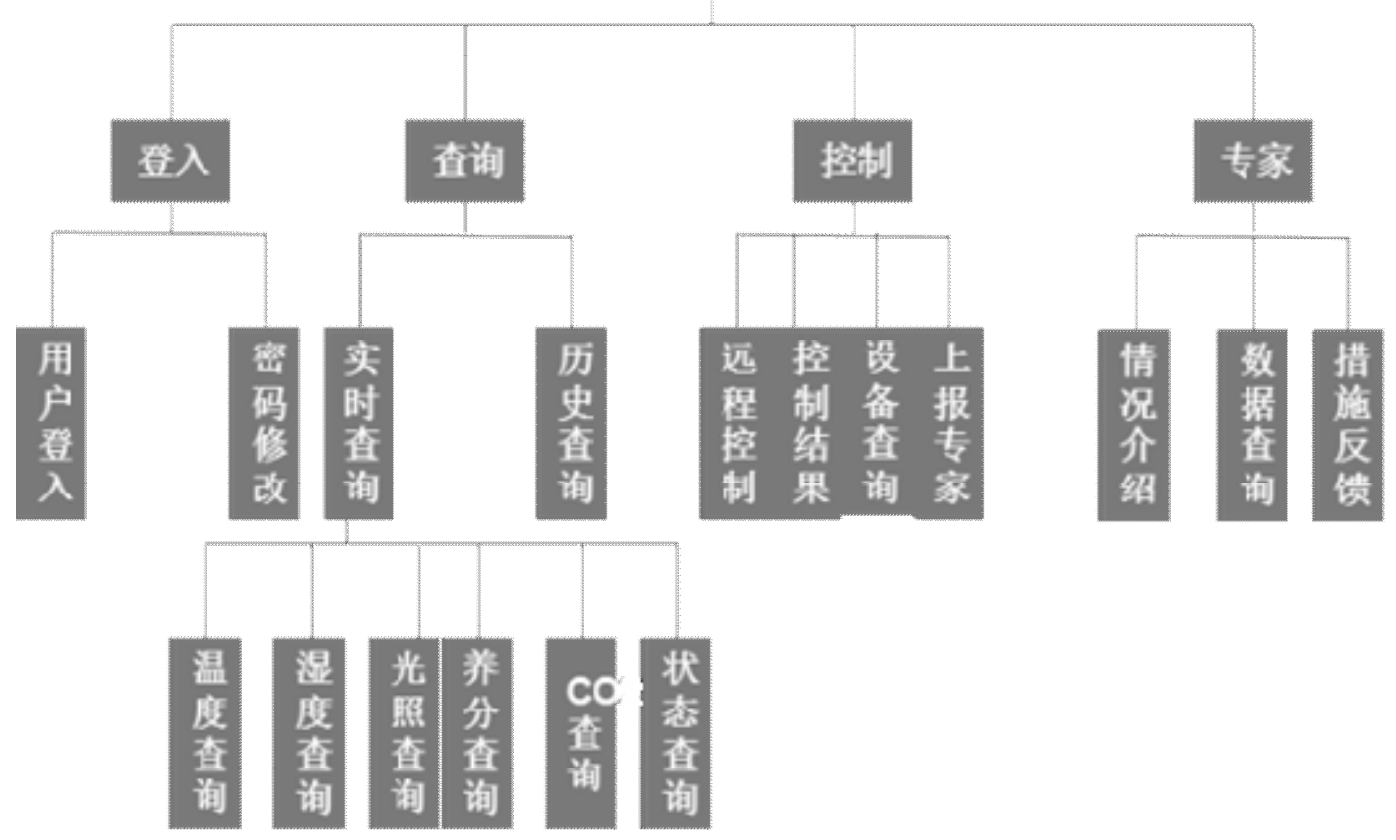

Fig.4 monitoring system function module diagram

Greenhouse monitoring system based on SSA \& D (or SDLC) method, in the Eclipse development environment combined with MySQL database, user needs as a criterion to gradually improve the 
system functions. Monitoring system with high reliability, high security, a high degree of modularity, through the cloud as a greenhouse and monitoring interface to communicate the bridge, the data transmission and instruction issued. Monitoring system control interface module is divided into four blocks: user management, query module, control module, expert advice module.

(1) user management module

Mainly for administrators, ordinary users, control users, experts and other roles for the definition and authorization of authority, as well as all users to delete or delete. For the sake of safety, the role of the user permissions set (1) administrator has the highest authority to manage all the roles of the authority (2) ordinary users can be all kinds of plants or some species of the growth of the query can not be controlled (3) control the user can For all or some species of plants to query and the environment for the most appropriate regulation (4) expert users to control the user reported abnormal phenomenon analysis proposed feedback to the control user

(2) query module

Real-time data query (1) temperature query: through the heat sensitive components to obtain accurate temperature, transfer data to the system, feedback to the query panel (2) humidity query, the use of humidity sensor for collection; (3) light query, through the light intensity measurement of light intensity; , Through the soil nutrient tester, accurate display of the main nutrients (nitrogen, phosphorus) content and $\mathrm{PH}$ value; (5) $\mathrm{CO} 2$ query, by the greenhouse carbon dioxide detector data transmission to the system. (6) growth status query, through remote remote control camera on the status of each plant for artificial inquiries to check the threat of pests. Historical data query, in order to show the form of plant near a period of time temperature, humidity, light, nutrients, $\mathrm{CO} 2$, and the recent control of the situation.

(3) control module

Each plant will pre-set the optimal range of its growth environment, the query display interface will prompt its appropriate area, when the range automatically "alarm" into the control interface, including real-time data, the appropriate range, the external environment, The current state of the equipment (in the case of awning, for example, the opening and closing of each awning, the opening and closing of the awning for how much). Based on this data, remote control of the awning, while testing whether the control data are in the appropriate range, due to light, temperature control will be applied to the awning, how to determine the opening and closing of awnings need to be based on follow-up environmental data.

\section{(4) Expert module}

When the control user can not determine the solution of the incident, the situation reported to the expert group, the expert group can be informed of the staff of the situation and the corresponding plant query interface and control interface, after the direct response to determine the program, the implementation of equipment control by the controller .

\section{Conclusions}

(1) Through the literature search and field research, based on the existing greenhouse monitoring system research, based on the establishment of the Internet of things greenhouse mobile terminal monitoring system. Intelligent monitoring and automatic control of the greenhouse, through the quality of the framework of mass heterogeneous data collection and processing to meet the needs of each crop of precision control.

(2) based on the Android platform for system design, reduce the difficulty of design and development and development costs, while simple operation has a general advantage. With the development of 
mobile data and wireless networks, monitoring can be done anytime, anywhere, to ensure timeliness and effectiveness.

The three modules of query, control and expert are designed to monitor the whole growth process in the process of crop growth and development and user management. For different types of different growth stages of the standard value set, by comparing the site environmental data values, the control program set, based on the most suitable environmental conditions of crops. But also "Internet of Things + Greenhouse + Android" three-dimensional model can not only be applied to crop growth monitoring, other agricultural products monitoring system has a reference.

\section{Acknowledgements}

This work was financially supported by the Shanghai Jiangsu Province students innovation and entrepreneurship training program (201610299045Z).

\section{References}

[1] Ahmad M. Nagib.SIGHTED: A Framework for Semantic Integration of Heterogeneous Sensor Data on the Internet of Things[J]. ScienceDirect ,2016:529-536.

[2] Li,Guohong.A Design of the IOT Gateway for Agricultural Greenhouse[J]. Sensors \& Transducers ,2013:75-80.

[3] JoséFrancisco Colom, Higinio Mora .Collaborative building of behavioural models based on internet of things[J]. Computers and Electrical Engineering,2016.

[4] Bahman Rashidi ,Carol Funga.Android fine-grained permission control system with real-time expert recommendations[J]. Pervasive and Mobile Computing,2016.

[5] Pawlowskia,J.A. Sánchez-Molinab.Evaluation of event-based irrigation system control scheme fortomato crops in greenhouses[J]. Agricultural Water Management,2016(7). 